\title{
Higher order mode analysis at the BNL Energy Recovery Linac
}

\author{
E.C. Johnson, I. Ben-Zvi, H. Hahn, L. Hammons, Wencan Xu
}

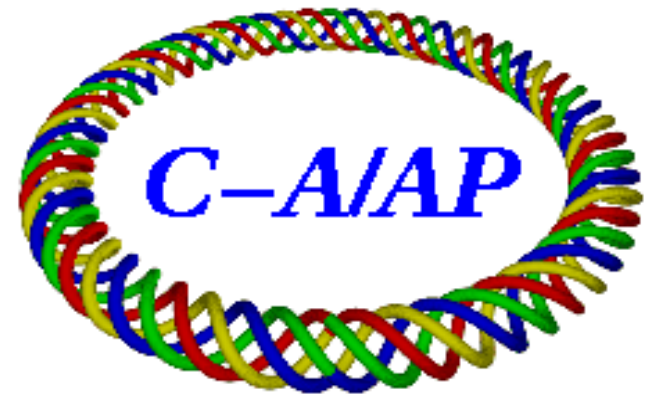

\section{Collider-Accelerator Department Brookhaven National Laboratory Upton, NY 11973}

Notice: This document has been authorized by employees of Brookhaven Science Associates, LLCunder Contract No. DE-AC02-98CH10886 with the U.S. Departm ent of En ergy. The United States Governm ent retains a nonexclusive, paid-up, irrevocable, world-wide license topublish or reproduce the published formof this document, or allow others to do so, for United States Government purposes. 


\title{
Higher-Order Mode Analysis at the BNL Energy Recovery Linac
}

\author{
E. C. Johnson*, I. Ben-Zvi, H. Hahn, L. Hammons, Wencan Xu \\ Accelerator RED Division, Brookhaven National Laboratory, Upton, NY 11973
}

(Dated: July 11, 2011)

\begin{abstract}
Understanding the prevalence and structure of higher-order modes (HOMs) in accelerator cavities is critical because their excitation can result in problematic single bunch and multi-bunch effects[1]. Particularly hazardous are dipole modes, which are more easily excited due to their linear field nature near the beam center. During a recent superconducting test on the energy recovery linac (ERL) cavity at Brookhaven National Laboratory (BNL), 8 of the highest-Q HOMs were measured for the first time. In conjunction with analysis of CST Microwave Studio simulation results for the ERL model, one of these modes was further studied in the copper prototype ERL cavity. A method of identifying HOMs utilizing existing holes drilled in copper cavity cells was developed and used to conclude that the observed high-Q mode was a quadrupole.
\end{abstract}




\section{INTRODUCTION}

An energy recovery linac (ERL) is a multi-pass accelerator that recirculates high-energy electrons out of phase in order to recover their energy with a high efficiency. Currently, an ampere-class superconducting ERL is under construction at the Collider-Accelerator Department at BNL, comprised of a 5-cell RF cavity and photo-injector RF electron gun, both operating at $703.75 \mathrm{MHz}[2]$. The facility is a proof-of-principle program serving as a test-bed for an envisioned luminosity upgrade for the Relativistic Heavy Ion Collider (RHIC) via electron cooling. This process involves injecting cold electrons into hot ion bunches, allowing Coulomb scattering to occur, then removing the electrons from the bunches. A schematic of the BNL ERL is given in Fig. 1.

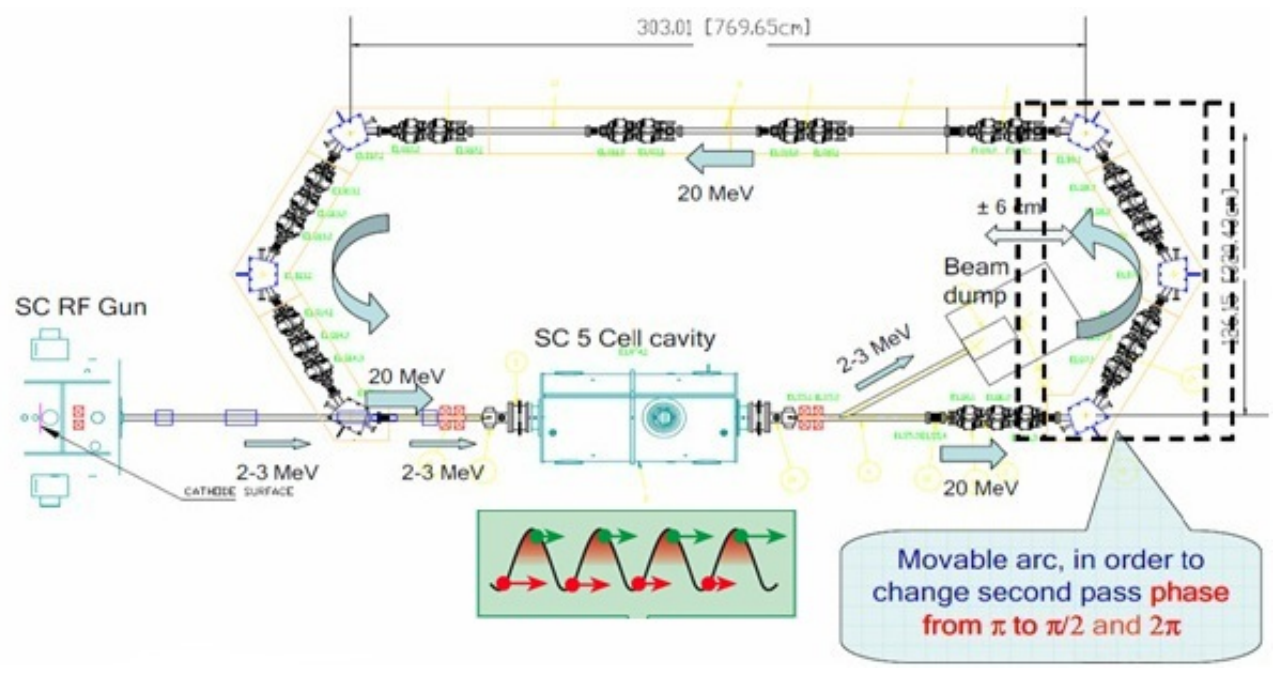

FIG. 1: Schematic of the ERL at BNL.

\section{Higher-Order Modes}

As in most resonators, accelerator cavities allow the occurrence of modes with propagation constants and transverse intensity profiles different from the design (or "fundamental") mode. These modes occur at higher frequencies than the fundamental mode, and are thus called higher-order modes $(\mathrm{HOMs})$. In an accelerator, the fundamental $\left(T M_{010}\right)$ mode has a longitudinal E-field along the cavity axis, which transfers energy in the RF field to kinetic energy in the particle bunch. However, the converse is also true: a charge bunch passing through an accelerator cavity can excite field patterns, and, in the case of off-axis charges, 
HOMs. These high-frequency resonances are detrimental to accelerator operation because they are a source of beam instabilities, and, in extreme cases, complete beam loss - a condition known as beam break-up (BBU). In superconducting systems, HOMs also result in additional cryogenic losses due to excessive power dissipation in cavity walls. To avoid these concerns, successful damping of HOMs is a critical requirement of accelerators.

\section{HOM Simulation with CST Microwave Studio}

The Collider-Accelerator Department at BNL uses CST Microwave Studio (MWS)[3] for electromagnetic analysis of accelerator components. This software employs a 3-dimensional CAD interface along with several different solvers, including eigenmode, transient, and frequency domain. The eigenmode solver is used to analyze HOMs, by outputting their frequency, field profiles, and $\frac{R}{Q}$ values (a quantity directly related to beam energy lost to the HOM - see Appendix). Fig. 2 shows the MWS model of the ERL cavity.

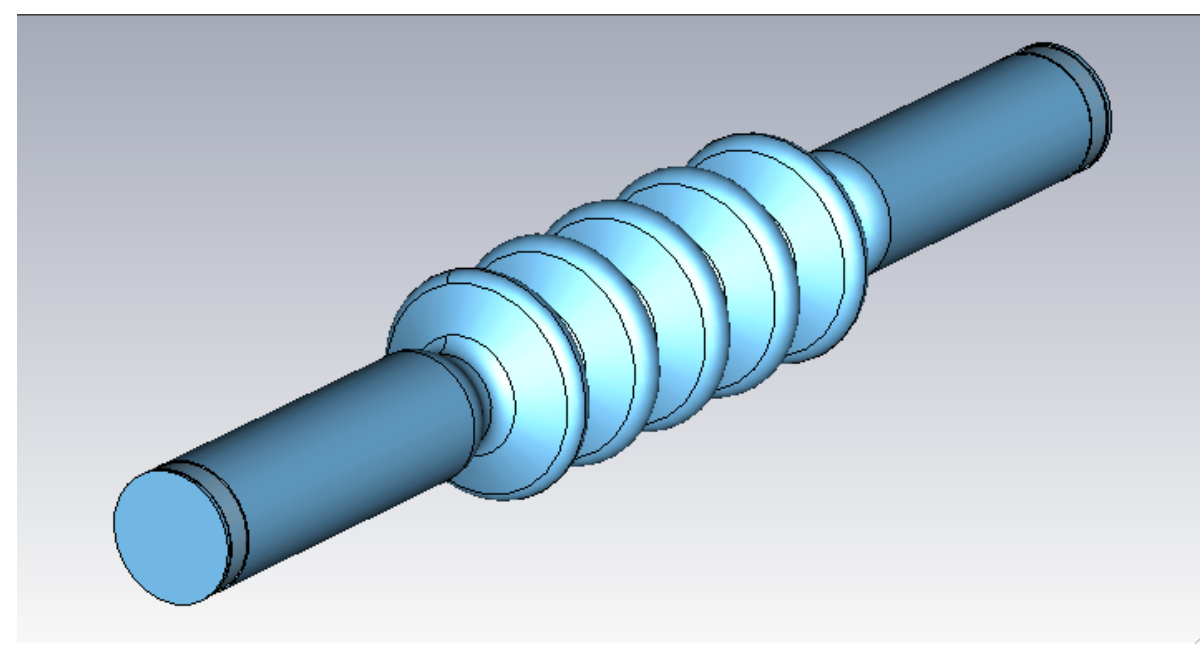

FIG. 2: MWS Model of the 5-cell BNL ERL cavity.

\section{HOM Measurement for the ERL}

To properly understand HOM behavior in the ERL cavity, comprehensive measurement and the resulting correspondence to MWS results are needed. Before construction of the niobium 5-cell cavity, 2 copper prototype cavities were fabricated to study many properties of the cavity including field flatness and cavity-to-cavity "crosstalk". These cavities are also 
useful for HOM study, as their dimensions are identical to the niobium cavity. (It is worth noting, however, that copper-cavity resonance characteristics won't match the operating ERL's exactly due to the measurement at room temperature and fabrication errors that lead to slight differences between cavity geometries.) The copper prototype and niobium cryostat assembly are shown in Figs. 3-4.

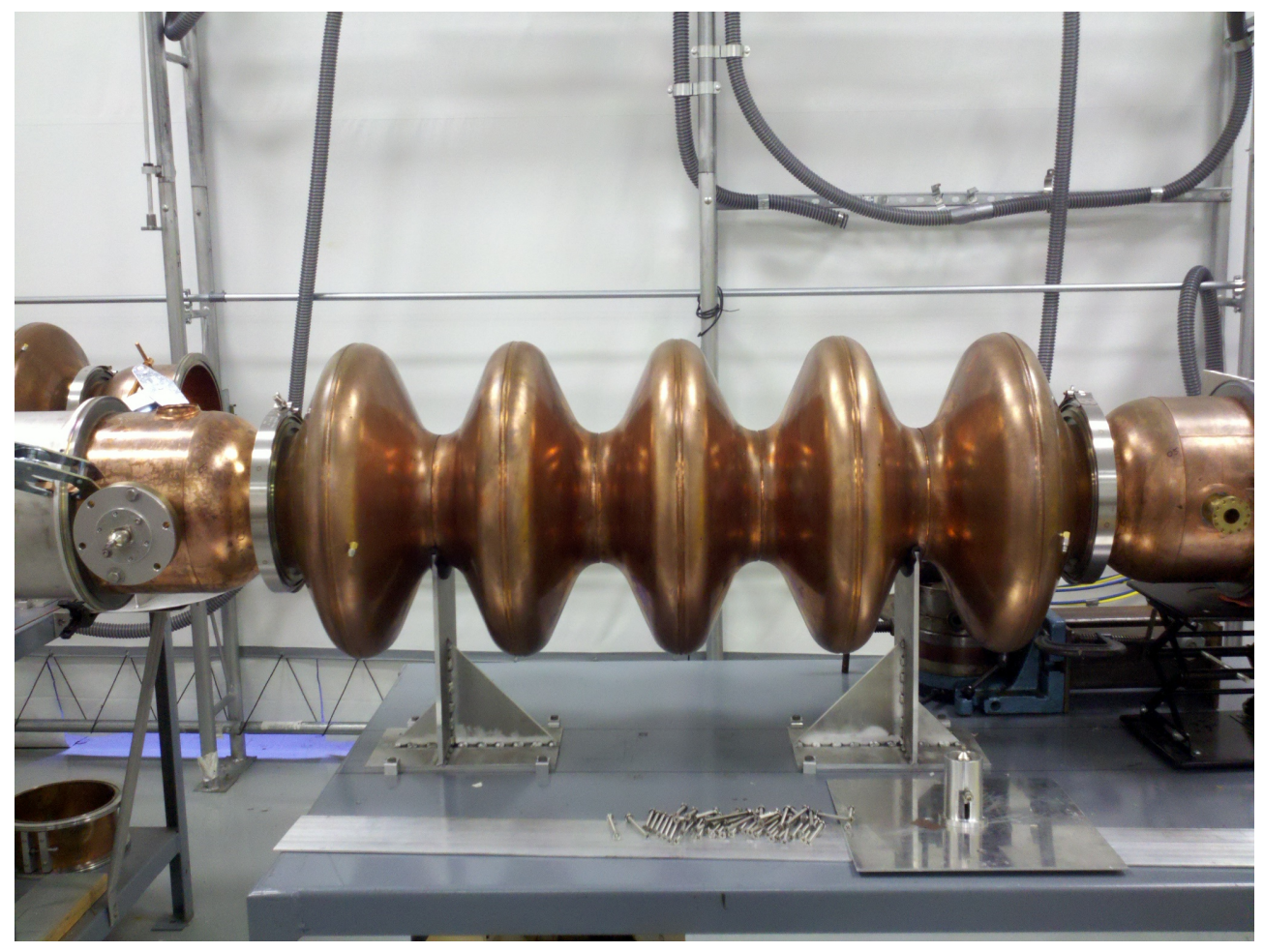

FIG. 3: Copper prototype cavity.

\section{COLD ERL MEASUREMENTS}

In March 2010, the ERL cavity was cooled to $4 K$ for RF testing. Before coupling power into the cavity, HOM measurements were made using the $S_{21}$ scheme of a network analyzer connected to the beam position monitor (BPM) ports on each end of the cavity (see Fig. 4). For this kind of measurement, the network analyzer inputs a signal in a BPM on one end of the cavity and detects transmission as a function of frequency on the other end. HOMs are observed as sharp transmission peaks, and characterized by their $Q$-value - a ratio of center frequency to peak width. During this measurement, there was no power being coupled into the cavity from the fundamental power coupler, so all the resonances seen are propagating 


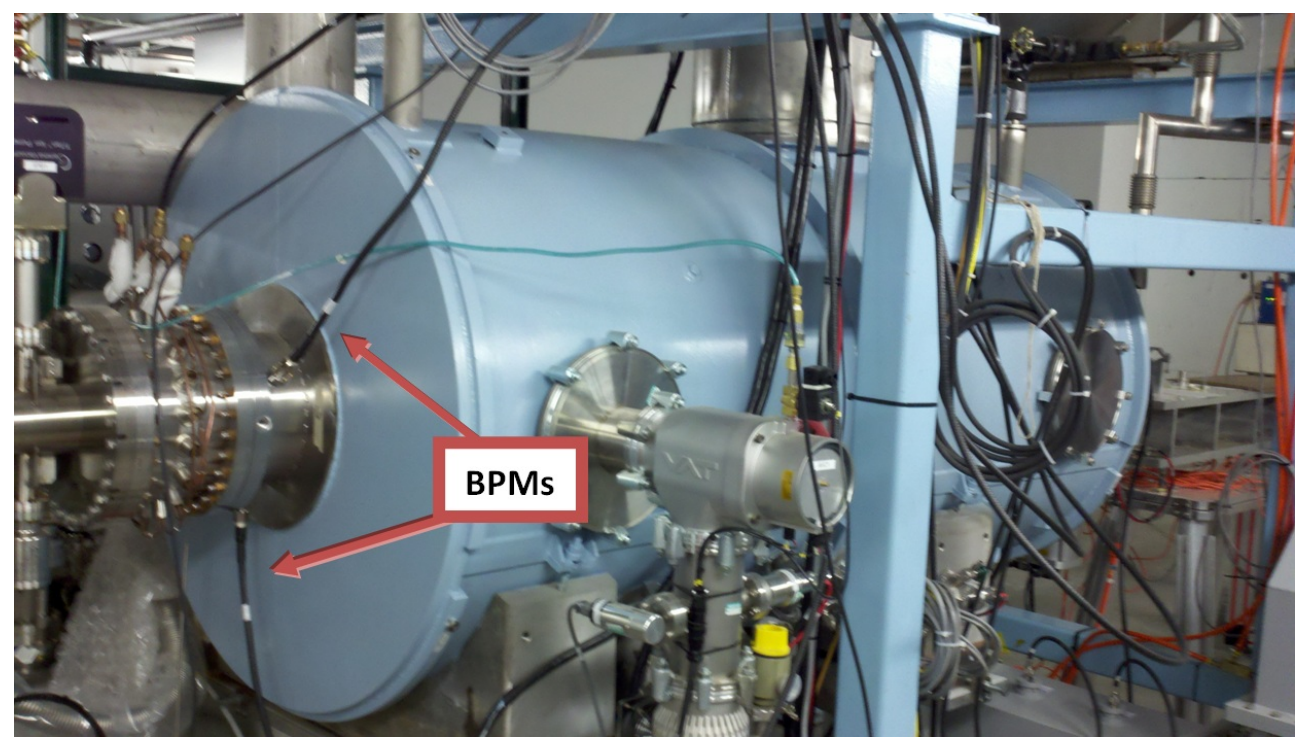

FIG. 4: ERL cryostat assembly. The beamtube is visible, along with 2 highlighted BPMs.

throughout the entire cavity. An example of a measured HOM resonance is shown in Fig. 5.

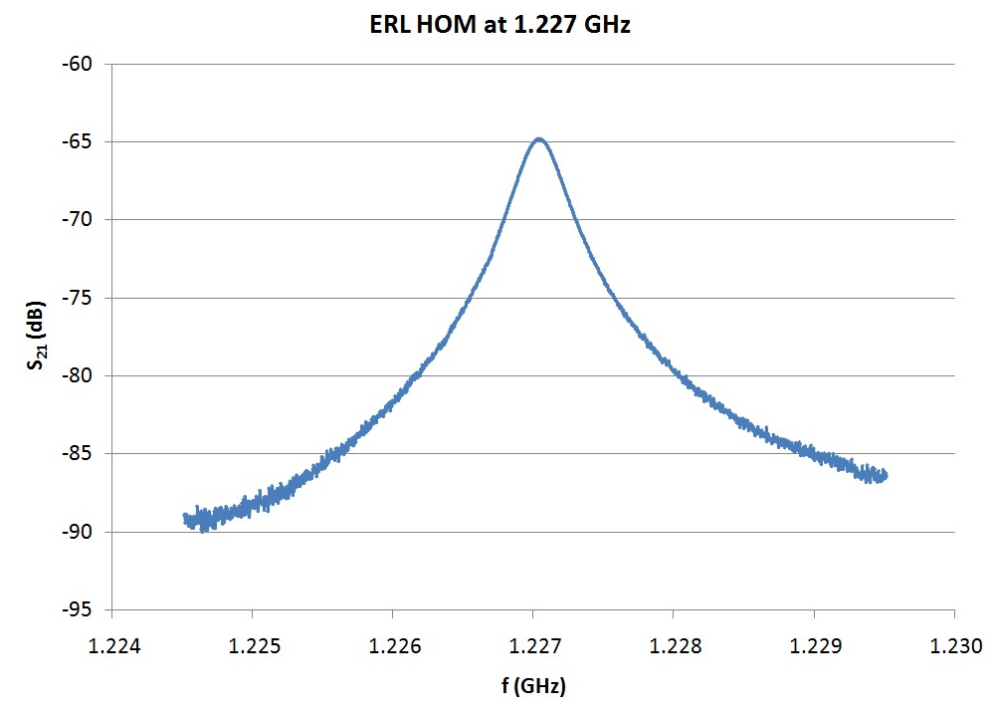

FIG. 5: HOM resonance measured in the ERL during a cold test.

A total of $8 \mathrm{HOMs}$ with Qs higher than any previously seen were measured, and are shown in Table I. Characterizing the structure (dipole, quadrupole, etc.) of the HOMs in Table I is critical, since dipoles with Q's of this order are expected to have shunt impedance values (see Appendix) nearing the BBU limit. A plot of the calculated BBU limit for dipoles in the ERL is shown in Fig. 6[4]. However, since the niobium ERL cavity is limited to 3 
TABLE I: Highest-Q HOMs measured in the ERL.

\begin{tabular}{||c|c||}
\hline$f(\mathbf{G H z})$ & $\mathbf{Q}$ \\
\hline 1.22008 & 920,000 \\
2.14764 & $5,503,500$ \\
2.14831 & $1,306,900$ \\
2.32984 & $2,291,800$ \\
2.34421 & 908,180 \\
2.45578 & $1,025,400$ \\
2.74324 & 620,040 \\
2.90829 & 406,090 \\
\hline
\end{tabular}

BPMs on each end, distinguishing between dipoles and any other HOM is impossible.

Alternatively, each cell of the copper prototype cavity has 8 holes ( $45^{\circ}$ apart) drilled around the outer perimeter. This number is large enough to distinguish HOM structure, so the copper cavity was used for a case study in identifying one of the HOMs from Table I $(f=1.22 \mathrm{GHz})$.

Beam Break-Up Limit for Dipoles in the ERL

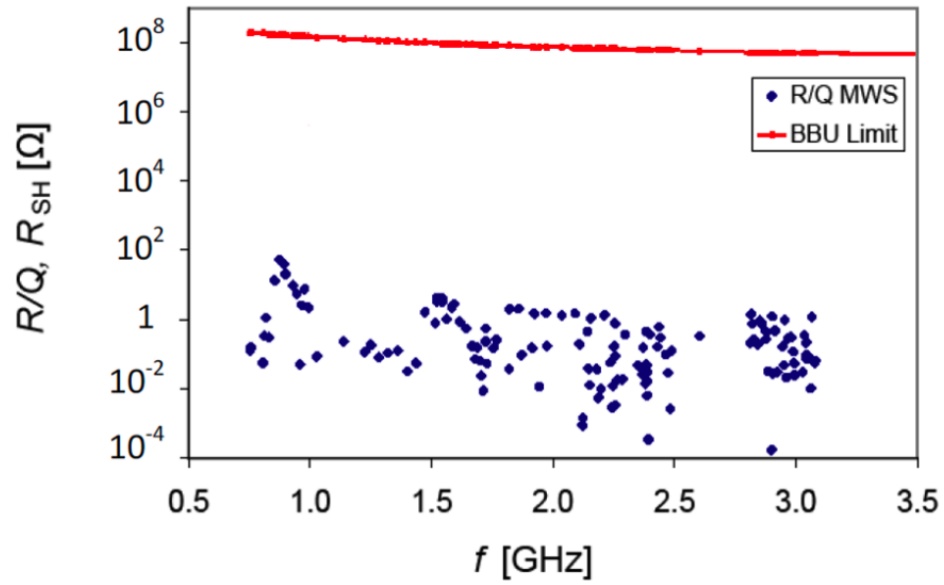

FIG. 6: Beam break-up limit for dipoles in the ERL.

Calculation courtesy of J. Kewisch 


\section{Copper Cavity Analysis of HOMs}

First, a correlation for the resonant nature between the cavities needed to be quantized in the $1.22 \mathrm{GHz}$ region. The correspondence is very good, as seen in Fig. 7. Some of the coppercavity modes in this plot exhibit mode "splitting", a condition where an asymmetry in the cavity (namely, a slightly elliptical rather than circular shape) causes 2 distinct polarizations of a mode to occur around the normal resonant frequency. The disparity between center frequencies for the HOMs between the cavities are shown in Table II. The center frequency for the split HOMs was used in the calculation. Between cavities, the frequencies of all modes change by less than $3 \mathrm{MHz}$, indicating a good correlation.

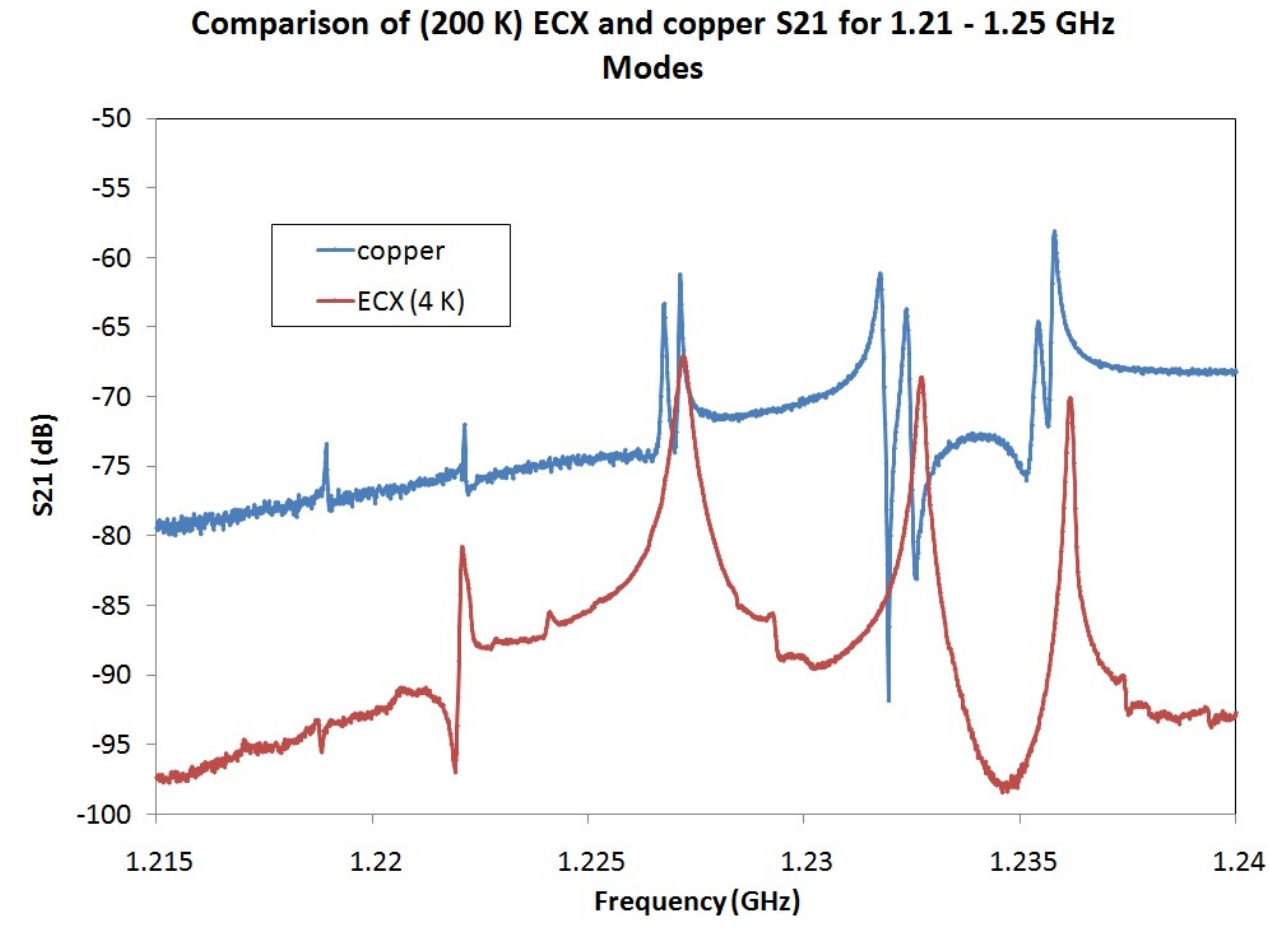

FIG. 7: Comparison of $\mathrm{Cu}$ - and Nb-cavity HOMs. A pass band of 5 resonances is seen, with a good correspondence between cavities.

To measure the HOM field structure, small conducting probes were made by stripping the outer layer of a coax line sized to the cell holes. One such probe tip is shown in Fig. 8. The arrangement of holes in the cells allows many amplitude and phase measurement configurations, both across cells or within one cell. The most elegant method for simply determining HOM structure is measuring the phase as a function of angle within a single cell. Observation of the azimuthal E-field behavior in an HOM show that the phase changes 
TABLE II: Comparison of Nb- and Cu-cavity HOMs in the 1.22 - $1.24 \mathrm{GHz}$ range.

\begin{tabular}{||c|c|c||}
\hline $\mathbf{N b} f(\mathbf{G H z})$ & $\mathbf{C u} f(\mathbf{G H z})$ & $\Delta(\mathbf{M H z})$ \\
$T=4 K$ & $T=300 K$ & \\
\hline 1.22008 & 1.21894 & 1.14 \\
1.22336 & 1.22215 & 1.21 \\
1.22859 & 1.22700 & 1.59 \\
1.23409 & 1.23195 & 2.14 \\
1.23748 & 1.23563 & 1.85 \\
\hline
\end{tabular}

by $180^{\circ}$ each time a node is crossed. This is further illustrated in Fig. 9. In the copper cavity, one of the 8 holes is needed for the excitation probe, so a total of $7 S_{21}$ measurements can be taken within the cell - enough to distinguish dipoles, quadrupoles, and sextupoles.
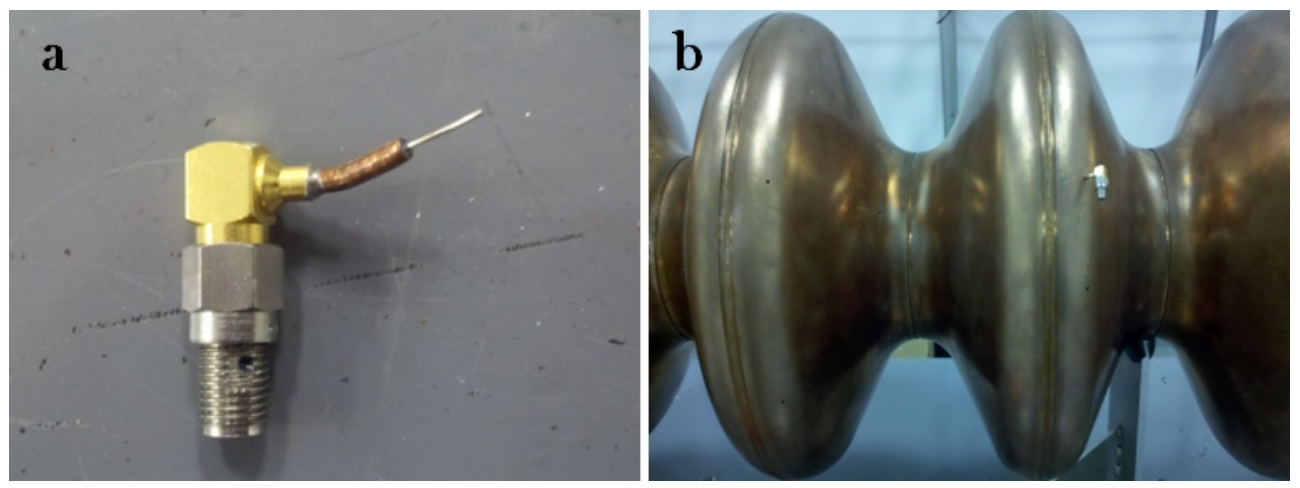

FIG. 8: a) Conducting probe for inter-cell copper cavity measurements. b) Probe inserted into cell for measurement.

\section{Result of Copper Cavity Measurement}

Using the inter-cell phase measurement method described above, the 5 HOMs shown in Fig. 7 were determined to be quadrupoles, as evidenced by $4180^{\circ}$ phase jumps. The results of these measurements are shown in Fig. 10.

These results compare favorably with the MWS analysis, which also indicated the occurrence of quadrupoles in this frequency range. The lowest-frequency MWS modes, occurring at 1.220 and $1.223 \mathrm{GHz}$, were seen to be "trapped" modes, with fields that do not propagate well into the beam tube (Fig. 11). By comparing $S_{21}$ measurements take in 2 configurations: 

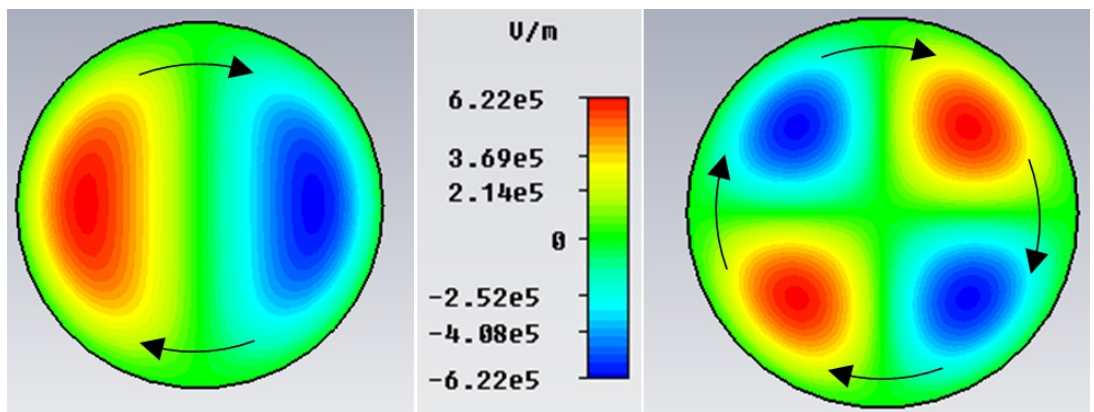

FIG. 9: Longitudinal field distribution in cavity cell for dipole and quadrupole modes. The opposite ends of the color spectrum denote the same field intensity but with a $180^{\circ}$ azimuthal phase change, so the arrows indicate crossing phase lines.
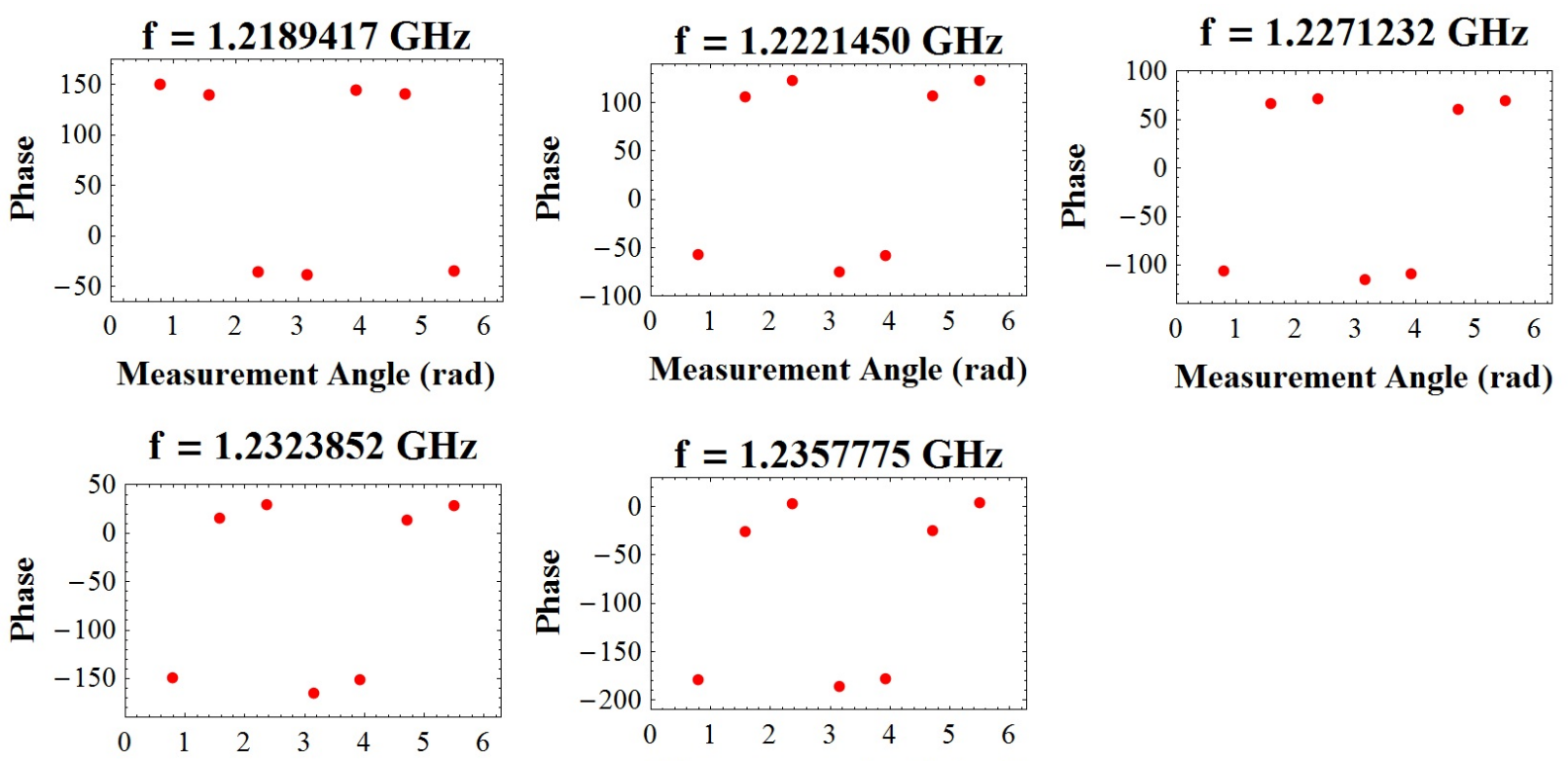

Measurement Angle (rad)

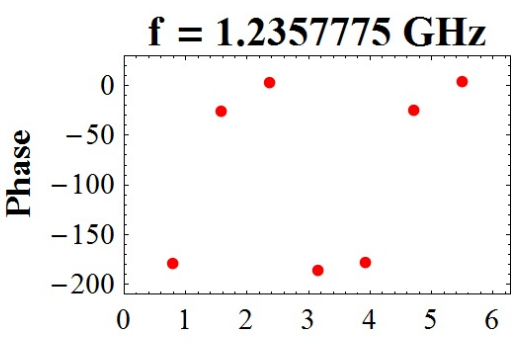

Measurement Angle (rad)

FIG. 10: Results of phase measurements, showing quadrupoles.

across the beamtubes and across outer cells, this same behavior was observed in the copper cavity, as shown in Fig. 12.

\section{CONCLUSION}

A high-current superconducting ERL is under construction at BNL. Recent $S_{21}$ measurements in the 5-cell ERL cavity at $4 K$ showed the prevalence of 8 previously unseen HOMs with $Q \approx 10^{6}$, a value large enough to induce BBU for dipole modes. A method of 


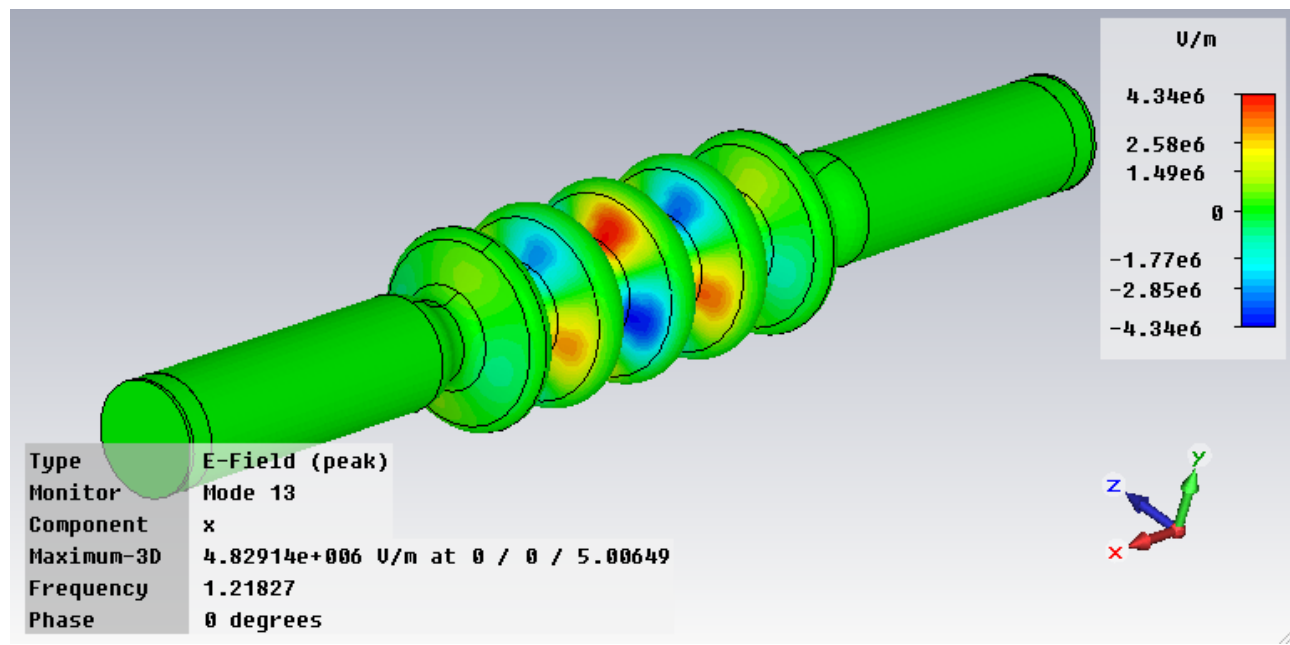

FIG. 11: Trapped quadrupole in MWS output.

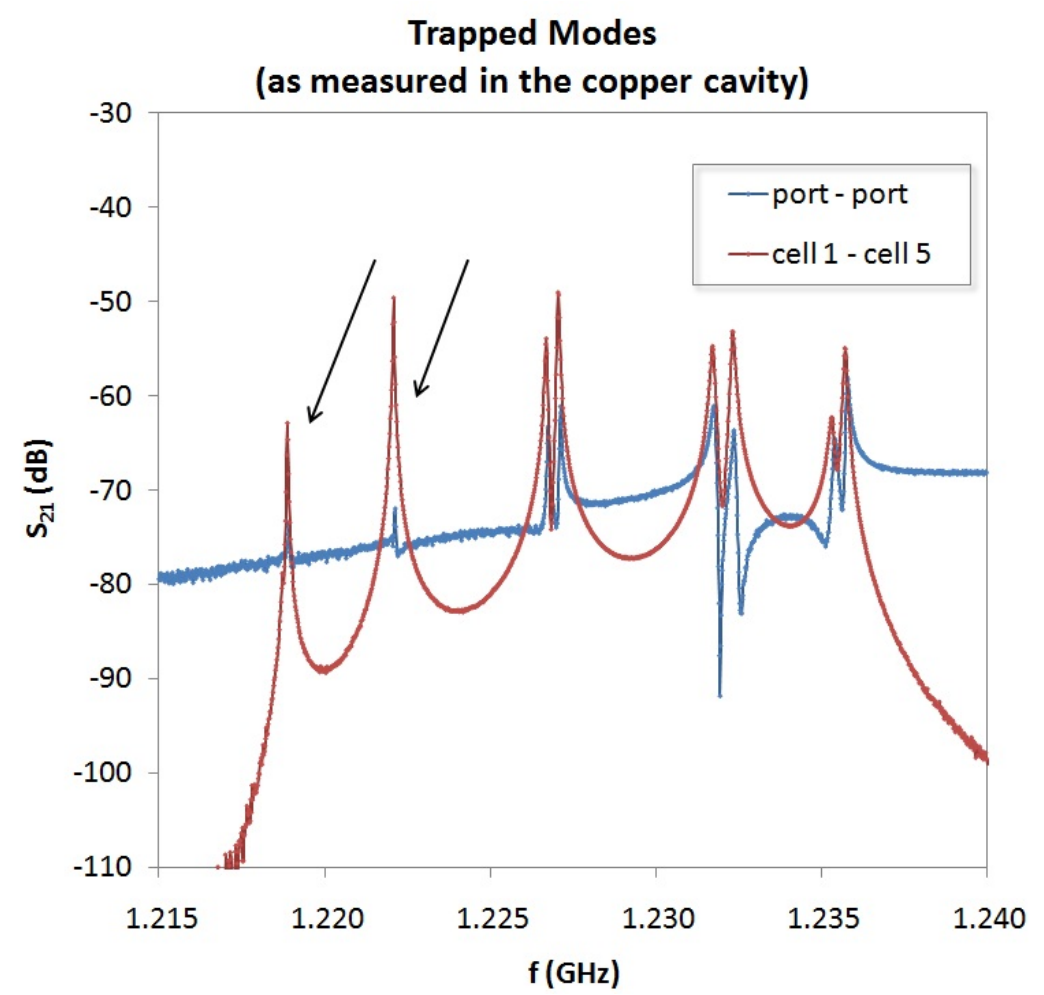

FIG. 12: Trapped modes measured in copper cavity, demonstrating a good correspondence with MWS.

identifying HOMs using a copper prototype ERL cavity was developed and used to study one of these HOMs, which was found to be a quadrupole. The result compared favorably with MWS simulation results. The HOM ID technique could be implemented on the other high-Q resonances measured in the ERL cavity. 


\section{APPENDIX}

The energy lost by a charge to a dipole mode is given by[5]:

$$
U_{q}=k_{d} q^{2}\left(\frac{\rho}{a}\right)^{2}
$$

where $q$ is the elementary charge, $a$ is the beam tube radius, $\rho$ is the distance off axis, and $k_{d}$ is the loss factor, which is directly related to a mode's "R over Q":

$$
k_{d}=a^{2}\left(\frac{\omega_{n}}{c}\right)^{2} \frac{\omega_{n}}{4} \frac{R_{s h}}{Q_{0}}
$$

Here $\omega_{n}$ is the mode's eigenfrequency, $c$ is the speed of light, $R_{s h}$ is the shunt impedance, and $Q_{0}$ is the quality factor. $Q_{0}$ is directly measured with a network analyzer as the ratio

of center frequency to resonance width. The value for $\frac{R_{s h}}{Q_{0}}$ is given as output in MWS. The product of $\frac{R_{s h}}{Q_{0}}$ and $Q_{0}$ is shunt impedance, a quantity which must be limited for beam stability, as shown in Fig. 6.

\section{REFERENCES}

1. R. Calaga, Ph.D. Thesis, Stony Brook University, 2006.

2. High Current Energy Recovery Linac at BNL, V.N. Litvinenko et al, in Proceedings of 2005 Particle Accelerator Conference, Knoxville, Tennessee

3. CST, http://www.cst.com.

4. Beam Break-Up Estimates for the ERL at BNL, I. Ben-Zvi et al, in Proceedings of IPAC'10, Kyoto, Japan

5. H. Padamsee, J. Knobloch, and T. Hays, RF Superconductivity for Accelerators, 2nd ed, (Wiley-VCH, 2008). 